\title{
An Enciphering Scheme Based on a Card Shuffle
}

\author{
Viet Tung Hoang ${ }^{1}$, Ben Morris $^{2}$, and Phillip Rogaway ${ }^{1}$ \\ 1 Dept. of Computer Science, \\ 2 Dept. of Mathematics, \\ University of California, Davis, USA
}

\begin{abstract}
We introduce the swap-or-not shuffle and show that the technique gives rise to a new method to convert a pseudorandom function (PRF) into a pseudorandom permutation (PRP) (or, alternatively, to directly build a confusion/diffusion blockcipher). We then prove that swap-or-not has excellent quantitative security bounds, giving a LubyRackoff type result that ensures security (assuming an ideal round function) to a number of adversarial queries that is nearly the size of the construction's domain. Swap-or-not provides a direct solution for building a small-domain cipher and achieving format-preserving encryption, yielding the best bounds known for a practical scheme for enciphering credit-card numbers. The analysis of swap-or-not is based on the theory of mixing times of Markov chains.
\end{abstract}

Keywords: Blockciphers, Feistel network, Luby-Rackoff, Markov chain, PRF-to-PRP conversion, pseudorandom permutations, swap-or-not.

\section{Introduction}

Overview. Despite the diversity of proposed blockciphers, only two approaches underlie the construction of real-world designs: essentially everything looks like some sort of Feistel network (e.g., DES, FEAL, MARS, RC6) or SP-network (e.g., Rijndael, Safer, Serpent, Square). Analogously, in the literature on constructing pseudorandom permutations (PRPs) from pseudorandom functions (PRFs), we have provable-security analyses for Feistel variants (e.g., [12 14, 18, 21]), as well as modes of operation (e.g., [10, 11, 18, 19]) that can again be construed as SP-networks, now on a large domain. Perhaps there just are not that many fundamentally different ways to make a blockcipher. Or perhaps we might have failed to notice other possibilities.

In this short paper we describe a very different way to make a blockcipher. We call it a swap-or-not network (or cipher or shuffle). Besides introducing the construction, we evidence its cryptographic utility. We do this by showing that swap-or-not provides the quantitatively best mechanism known, in terms of concrete security bounds, to convert a PRF into a PRP. We also show that swap-or-not provides a practical solution for the problem of format-preserving encryption (FPE) on domains of troublesome size, such as enciphering creditcard numbers. 


proc $E_{K F}(X)$
for $i \leftarrow 1$ to $r$ do
$\quad X^{\prime} \leftarrow K_{i} \oplus X$
$\quad \hat{X} \leftarrow \max \left(X, X^{\prime}\right)$
$\quad$ if $F_{i}(\hat{X})=1$ then $X \leftarrow X^{\prime}$
return $X$

Fig. 1. Cipher $E=\mathrm{SN}[r, n]$ encrypts $X \in\{0,1\}^{n}$ using a key $K F$ naming $K_{1}, \ldots, K_{r} \in\{0,1\}^{n}$ and round functions $F_{1}, \ldots, F_{r}:\{0,1\}^{n} \rightarrow\{0,1\}$
Construction. Suppose we aim to encipher $n$-bit strings; our message space is the set $\mathcal{X}=\{0,1\}^{n}$. Assume we will use $r$ rounds, and that the blockcipher's key $K F$ names subkeys $K_{1}, \ldots, K_{r} \in\{0,1\}^{n}$ as well as round functions $F_{1}, \ldots, F_{r}$, each of which maps $n$-bits to a single bit, so $F_{i}:\{0,1\}^{n} \rightarrow\{0,1\}$. Then we encipher $X \in\{0,1\}^{n}$ as shown in Fig. 1. The reason that this works, that one gets a permutation, is simply that $X \mapsto K_{i} \oplus X$ is an involution, and our round function depends on the set $\left\{X, K_{i} \oplus X\right\}$. The inverse direction for swap-or-not is identical to the forward direction shown above except for having $i$ run from $r$ down to 1 .

Restating the algorithm in English, at each round $i$ we pair the current value of $X \in\{0,1\}$ " with a "partner" point $X^{\prime}=K_{i} \oplus X$. We either replace $X$ by its partner or leave it alone. Which of these two things we do is determined by applying the boolean-valued $F_{i}$ to the two-element set $\left\{X, X^{\prime}\right\}$. Actually, in order to give $F_{i}$ a more conventional domain, we select a canonical representative from $\left\{X, X^{\prime}\right\}$, say $\hat{X}=\max \left(X, X^{\prime}\right)$, and apply $F_{i}$ to it. Note that each plaintext maps to a ciphertext by xoring into it some subset of the subkeys $\left\{K_{1}, \ldots, K_{r}\right\}$. This might sound linear, but it most definitely is not.

CARD SHufFling VIEW. The swap-or-not construction was invented, and will be analyzed, by regarding it as a way to shuffle a deck of cards. Seeing a blockcipher as a card shuffle enables one to exploit a large body of mathematical techniques, these dating back to the first half of the twentieth century. In addition, some ways to shuffle cards give rise to enciphering schemes that cryptographers did not consider. Swap-or-not is such a case.

One can always see a card shuffle as an enciphering scheme, and vice versa. If you have some method to shuffle $N$ cards, this determines a corresponding way to encipher $N$ points: place a card at each position $X \in[N]$, where $[N]=$ $\{0,1, \ldots, N-1\}$; shuffle the deck; then look to see the position where the card initially at position $X$ ended up. Call that position the ciphertext $Y$ for $X$. The randomness used in the shuffle corresponds the cipher's key.

The first thing needed for a card shuffle to give rise to a computationally feasible blockcipher is that the shuffle be oblivious, an idea suggested by Moni Naor [18, p. 62], 23, p. 17]. In an oblivious shuffle one can trace the trajectory of a card without attending to lots of other cards in the deck. Most conventional shuffles, such as the riffle shuffle, are not oblivious. The Thorp shuffle [26] is oblivious - and so is swap-or-not. As a shuffle, here's how it looks. 
Recasting swap-or-not as a way to shuffle cards, suppose we have $N$ cards, one at each position $X \in[N]$, where $N=2^{n}$. To shuffle the deck, choose a random $K \in\{0,1\}^{n}$ and then, for each pair of card positions $X$ and $K \oplus X$, flip a fair coin. If it lands heads, swap the cards at the indicated positions; if it lands tails, leave them alone. See Fig. 2. The process can be repeated any number $r$ times, using independent coins (both the $K$-values and the $b$-values) for each shuffle.

When the swap-or-not shuffle of Fig. 2 is translated back into the language of encryption, one recovers the swap-or-not cipher of Fig. 1; these are different views of precisely the same process. The random pairing-up of cards specified by $K$ for the $i$ th shuffle corresponds to the subkey $K_{i}$. The random bit $b$ flipped at the shuffle's round $i$ for the pair $\{X, K \oplus X\}$ corresponds $F_{i}(\hat{X})$.

proc $E_{K F}(X)$
for $i \leftarrow 1$ to $r$ do
$\quad X^{\prime} \leftarrow K_{i}-X$
$\quad \hat{X} \leftarrow \max \left(X, X^{\prime}\right)$
$\quad$ if $F_{i}(\hat{X})=1$ then $X \leftarrow X^{\prime}$
return $X$

Fig. 3. Cipher $E=\mathrm{SN}[r, N,+]$ encrypts $X \in[N]$ using a key $K F$ naming $K_{1}, \ldots, K_{r} \in[N]$ and round functions $F_{1}, \ldots, F_{r}:[N] \rightarrow\{0,1\}$ a power of two - we'll be able to encipher points on any set $\mathcal{X}=[N]$, just by naming a group operator, say addition modulo $N$. For generalizing the shuffle of Fig. 2, the value $K$ is uniformly drawn from $[N]$ rather than from $\{0,1\}^{n}$, and we consider the pair of positions $\{X, K-X\}$ rather than $\{X, K \oplus X\}$. For the generalized cipher - see Fig. 3 - the key $K F$ will name subkeys $K_{1}, \ldots, K_{r} \in[N]$ and round functions $F_{1}, \ldots, F_{r}:[N] \rightarrow\{0,1\}$. We set $X^{\prime} \leftarrow K_{i}-X$ rather than $X^{\prime} \leftarrow K_{i} \oplus X$. The inverse remains what one gets by iterating from $r$ down to 1 .

Results. As with Luby and Rackoff's seminal paper [14], we can analyze the swap-or-not construction by regarding its constituent parts as uniformly random. Formally, let us write $\mathrm{SN}[r, N,+]: \mathcal{K} \times[N] \rightarrow[N]$ for the blockcipher $E$ specified in Fig. 3 that is swap-or-not with $r$ rounds, a message space of $[N]$, the indicated group operator, and where the key space names all possible subkeys $K_{1}, \ldots, K_{r} \in[N]$ and all possible round functions $F_{1}, \ldots, F_{r}:[N] \rightarrow\{0,1\}$. 


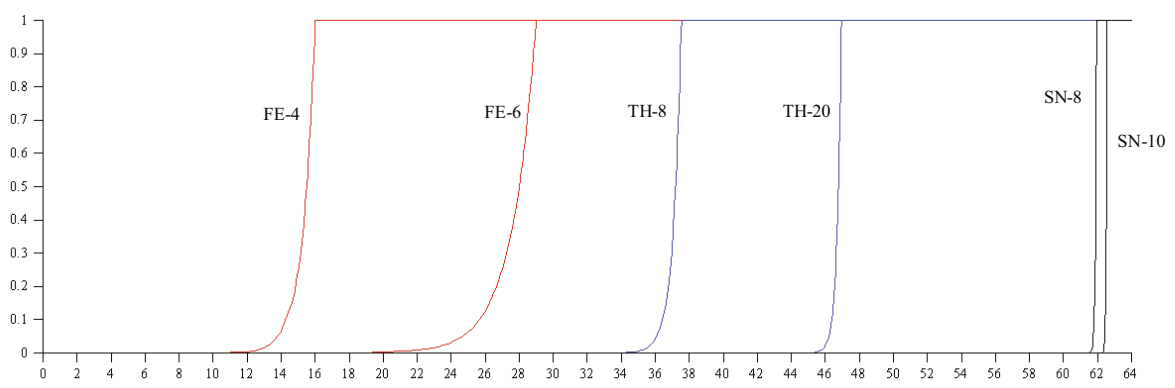

Fig. 4. Illustration of results. The message space has $N=2^{64}$ points. The graphs show established upper bounds on CCA advantage when the adversary asks $q$ queries, where $\log _{2}(q)$ labels the $x$-axis. Rightmost two graphs: the new results - the swap-or-not cipher for either eight passes (512 rounds) (SN-8) or 10 (SN-10), as given by Theorem 4 (One pass is defined as $\lceil\lg N\rceil$ rounds.) For comparison, the leftmost two graphs are for balanced Feistel, both the classical 4-round result of Luby and Rackoff [14, 20] (LR-4) and then a six-round result of Patarin (LR-6) [22, Th. 7]. The middle two graphs are for the Thorp shuffle, either with eight passes (TH-8) or 20 (TH-20), as given by [17, Th. 5].

Thus a random key $K F$ for this cipher has the $K_{i}$ and $F_{i}$ values uniformly chosen. We define the CCA (also called the "strong-PRP") advantage of an adversary $A$ attacking $E$ by dropping it into one of two worlds. In the first, the adversary gets an oracle for $E_{K F}(\cdot)$, for a random $K F$, and also an oracle for its inverse, $E_{K F}^{-1}(\cdot)$. Alternatively, the adversary is given a uniformly random permutation $\pi:[N] \rightarrow[N]$, along with its inverse, $\pi^{-1}(\cdot)$. Define

$$
\mathbf{A d v}_{\mathrm{SN}[r, N,+]}^{\mathrm{cca}}(q)=\max _{A}\left\{\operatorname{Pr}\left[A^{E_{K F}(\cdot), E_{K F}^{-1}(\cdot)} \Rightarrow 1\right]-\operatorname{Pr}\left[A^{\pi(\cdot), \pi^{-1}(\cdot)} \Rightarrow 1\right]\right\},
$$

the maximum over all adversaries that ask at most $q$ total queries. Our main result is that

$$
\operatorname{Adv}_{\mathrm{SN}[r, N,+]}^{\mathrm{cca}}(q) \leq \frac{4 N^{3 / 2}}{r+4}\left(\frac{q+N}{2 N}\right)^{r / 4+1}
$$

Roughly said, you need $r=6 \lg N$ rounds of swap-or-not to start to see a good bound on CCA-security. After that, the adversary's advantage drops off inverse exponentially in $r$. The summary explanation of formula (1) just given assumes that the number of adversarial queries is capped at $q=(1-\epsilon) N$ for some fixed $\epsilon>0$.

The quantitative guarantee above is far stronger than anything a balanced Feistel network can deliver. The only remotely comparable bound we know, retaining security to $N^{1-\epsilon}$ queries instead of $(1-\epsilon) N$ queries, is the Thorp shuffle [26] (or, equivalently, a maximally-unbalanced Feistel network 17]). But the known result, establishing $\mathbf{A d v}_{E^{\prime}}^{\text {cca }}(q) \leq(2 q / r+1)(4 n q / N)^{r}$ if one shuffles 
$N=2^{n}$ points for $r(4 n-2)$ rounds [17], vanishes by the time that $q \geq \frac{N}{4 \lg N}$. Numerically, the Thorp-shuffle bounds come out much weaker for most $r, q$, and $N$. See Fig. 4 for sample graphs comparing known bounds on balanced Feistel, the Thorp shuffle, and swap-or-not.

As a simple numerical example, swap-or-not enciphering 64-bit strings for 1200 rounds using a random round function will yield a maximal CCA advantage of less than $10^{-10}$, even if the adversary can ask $q=2^{63}$ queries. While the number of rounds is obviously large, no other construction can deliver a comparable guarantee, achieving security even when $q$ is close to $N$.

For a more complexity-theoretic discussion of swap-or-not, see Section 4 ,

FORMAT-PRESERVING ENCRYPTION. Swap-or-not was originally invented as a solution for format-preserving encryption (FPE) [1, 3, 5], where it provides the best known solution, in terms of proven-security bounds, when $N$ is too big to spend linear time computing, yet too small for conventional constructions to deliver desirable bounds. This landscape has not much changed with the recent work of Stefanov and Shi 24], who, following Granboulan and Pornin [9], show how to speed up (e.g., to $\tilde{\Theta}\left(N^{0.5}\right)$ time) determining where a card goes in a particular $N$-card shuffle after spending $\tilde{\Theta}(N)$ time at key-setup. For more discussion of swap-or-not and its use in FPE, see Section 5 .

\section{Preliminaries}

TOtal VARIATION DisTANCE. Let $\mu$ and $\nu$ be probability distributions on $\Omega$. The total variation distance between distributions $\mu$ and $\nu$ is defined as

$$
\|\mu-\nu\|=\frac{1}{2} \sum_{x \in \Omega}|\mu(x)-\nu(x)|=\max _{S \subset \Omega}\{\mu(S)-\nu(S)\} .
$$

BlockCiphers. Let $E: \mathcal{K} \times \mathcal{M} \rightarrow \mathcal{M}$ be a blockcipher, meaning that $\mathcal{K}$ and $\mathcal{M}$ are finite and each $E_{K}(\cdot)=E(K, \cdot)$ is a permutation on $\mathcal{M}$. We emphasize that $\mathcal{K}$ and $\mathcal{M}$ need not consist of binary strings of some particular length, as is often assumed to be the case. For any blockcipher $E$, we let $E^{-1}$ be its inverse blockcipher.

For blockcipher $E: \mathcal{K} \times \mathcal{M} \rightarrow \mathcal{M}$ and adversary $A$ the advantage of $A$ in carrying out an (adaptive) chosen-ciphertext attack (CCA) on $E$ is

$$
\operatorname{Adv}_{E}^{\text {cca }}(A)=\operatorname{Pr}\left[K \stackrel{\$}{\leftarrow} \mathcal{K}: A^{E_{K}(\cdot), E_{K}^{-1}(\cdot)} \Rightarrow 1\right]-\operatorname{Pr}\left[\pi \stackrel{\$}{\leftarrow} \operatorname{Perm}(\mathcal{M}): A^{\pi(\cdot), \pi^{-1}(\cdot)} \Rightarrow 1\right] .
$$

Here $\operatorname{Perm}(\mathcal{M})$ is the set of all permutations on $\mathcal{M}$. We say that $A$ carries out an (adaptive) chosen-plaintext attack (CPA) if it asks no queries to its second oracle. Adversary $A$ is non-adaptive if it asks the same queries on every run. Let $\operatorname{Adv}_{E}^{\text {cca }}(q)$ be the maximum advantage of any (adaptive) CCA adversary against $E$ subject to the adversary asking at most $q$ total oracle queries. Similarly define $\mathbf{A d v}_{E}^{\text {ncpa }}(q)$ for nonadaptive CPA attacks (NCPA). 
For blockciphers $F, G: \mathcal{K} \times \mathcal{M} \rightarrow \mathcal{M}$ let $F \circ G$ denote their cascade, with $F$ 's output fed into $G$ 's input; formally, $F \circ G: \mathcal{K}^{2} \times \mathcal{M} \rightarrow \mathcal{M}$ is defined by $(F \circ G)_{\left(K, K^{\prime}\right)}=G_{K^{\prime}}\left(F_{K}(X)\right)$.

LIFTING NCPA TO CCA SECURITY. We bound the CCA-security of a Feistel network from its NCPA-security by using the following result of Maurer, Pietrzak, and Renner [15, Corollary 5]. It is key to our approach, effectively letting us assume that our adversaries are of the simple, NCPA breed. Recall that in writing $F \circ G$, the blockciphers are, in effect, independently keyed.

Lemma 1 (Maurer-Pietrzak-Renner). If $F$ and $G$ are blockciphers on the same message space then, for any $q, \mathbf{A d v}_{F \circ G^{-1}}^{\text {cca }}(q) \leq \mathbf{A d v}_{F}^{\text {ncpa }}(q)+\mathbf{A d}_{\mathbf{d}}^{\text {ncpa }}(q)$.

\section{Security of Swap-or-Not}

Fix a finite abelian group $G=([N],+)$ where $[N]=\{0,1, \ldots, N-1\}$. We define the swap-or-not shuffle $\mathrm{SN}[r, N,+]$ of $r$ rounds over the elements of $G$. The shuffling at round $t$ is as follows. Initially, each of $N$ distinct cards is at a position in the set $[N]$. To shuffle during this round, choose $K_{t} \stackrel{\$}{\leftarrow}[N]$, the subkey at round $t$. Then, for each set $\left\{X, K_{t}-X\right\}$ with $X \in G$, choose $b \stackrel{\$}{\leftarrow}\{0,1\}$ and then swap the cards at positions $X$ and $K_{t}-X$ if $b=1$.

Let $\left\{W_{t}: t \geq 0\right\}$ be the Markov chain representing the swap-or-not shuffle with $N$ cards. More formally, let $\mathcal{C}$ be a set of cardinality $N$, whose elements we call cards. The state space of $\left\{W_{t}\right\}$ is the set of bijections from $\mathcal{C}$ to $\{0, \ldots, N-1\}$. For a card $z \in \mathcal{C}$, we interpret $W_{t}(z)$ as the position of card $z$ at time $t$.

Let $A$ be a deterministic adversary that makes exactly $q$ queries. Our proof is based on an analysis of the mixing rate of the swap-or-not shuffle. However, since $A$ makes only $q \leq N$ queries, we need only bound the rate at which some $q$-element subset of the cards mixes. So let $z_{1}, \ldots, z_{q}$ be distinct cards in $\mathcal{C}$, and let $X_{t}$ be the vector of positions of cards $z_{1}, \ldots, z_{q}$ at time $t$. For $j$ in $\{1, \ldots, q\}$ we write $X_{t}(j)$ for the position of card $z_{j}$ at time $t$, and define $X_{t}(1, \ldots, j)=\left(X_{t}(1), \ldots, X_{t}(j)\right)$. We shall call $X_{t}$ the projected swap-or-not shuffle. Note that the stationary distribution of $X_{t}$, which we denote by $\pi$, is uniform over the set of distinct $q$-tuples of elements from $G$. Equivalently, $\pi$ is the distribution of $q$ samples without replacement from $G$. Let $\tau_{t}$ denote the distribution of $X_{t}$.

Theorem 2 (Rapid mixing). Consider the swap-or-not shuffle $\operatorname{SN}[r, N,+]$ for $r, N \geq 1$, and let $q \in\{1, \ldots, N\}$. Fix $z_{1}, \ldots, z_{q}$ and let $\left\{X_{t}: t \geq 0\right\}$ be the corresponding projected swap-or-not shuffle, let $\pi$ be its stationary distribution, and let $\tau_{t}$ be the distribution of $X_{t}$. Then

$$
\left\|\tau_{r}-\pi\right\| \leq \frac{2 N^{3 / 2}}{r+2}\left(\frac{q+N}{2 N}\right)^{r / 2+1}
$$


Proof. Let $\tau_{t}^{k}$ be the conditional distribution of $X_{t}$ given the subkeys $K_{1}, \ldots, K_{r}$. (Here we consider $K_{1}, \ldots, K_{r}$ random variables, and we condition on the $\sigma$ algebra of these random variables.) We will actually show that $\mathbf{E}\left(\left\|\tau_{r}^{k}-\pi\right\|\right)$ satisfies the claimed inequality. Note that since $K_{1}, \ldots, K_{r}$ are random variables, so is $\tau_{r}^{k}$, and hence so is $\left\|\tau_{r}^{k}-\pi\right\|$. This implies the theorem since $\tau_{r}=\mathbf{E}\left(\tau_{r}^{k}\right)$ and hence

$$
\left\|\tau_{r}-\pi\right\|=\left\|\mathbf{E}\left(\tau_{r}^{k}-\pi\right)\right\| \leq \mathbf{E}\left(\left\|\tau_{r}^{k}-\pi\right\|\right)
$$

by Jensen's inequality, since for distributions $\mu$ and $\tau$, the total variation distance $\|\mu-\tau\|$ is half the $L^{1}$-norm of $\mu-\tau$, and the $L^{1}$-norm is convex. For a distribution $\nu$ on $q$-tuples of $\Omega$, define

$$
\begin{aligned}
\nu\left(u_{1}, \ldots, u_{j}\right) & =\operatorname{Pr}\left[Z_{1}=u_{1}, \ldots, Z_{j}=u_{j}\right] \text { and } \\
\nu\left(u_{j} \mid u_{1}, \ldots, u_{j-1}\right) & =\operatorname{Pr}\left[Z_{j}=u_{j} \mid Z_{1}=u_{1}, \ldots, Z_{j-1}=u_{j-1}\right]
\end{aligned}
$$

where $\left(Z_{1}, \ldots, Z_{q}\right) \sim \nu$. For example, $\tau_{t}\left(u_{1}, \ldots, u_{j}\right)$ is the probability that, in the swap-or-not shuffle, cards $z_{1}, \ldots, z_{j}$ land in positions $u_{1}, \ldots, u_{j}$ at time $t$, while $\tau_{t}\left(u_{j} \mid u_{1}, \ldots, u_{j-1}\right)$ is the probability that at time $t$ card $z_{j}$ is in position $u_{j}$ given that cards $z_{1}, \ldots, z_{j-1}$ are in positions $u_{1}, \ldots, u_{j-1}$. On the other hand, $\pi\left(u_{j} \mid u_{1}, \ldots u_{j-1}\right)$ is the probability that, in a uniform random ordering, card $z_{j}$ is in position $u_{j}$ given that cards $z_{1}, \ldots, z_{j-1}$ land in positions $u_{1}, \ldots, u_{j-1}$.

Each of the conditional distributions $\tau_{t}^{k}\left(\cdot \mid u_{1}, \ldots, u_{j-1}\right)$ converges to uniform as $t \rightarrow \infty$. When all of these distributions are "close" to uniform, then $\tau_{t}^{k}$ will be close to $\pi$. In fact, we only need the conditional distributions to be close "on average," as is formalized in the following lemma, which is easily established using coupling. For a proof, see [17, Appendix A].

Lemma 3. Fix a finite nonempty set $\Omega$ and let $\mu$ and $\nu$ be probability distributions supported on $q$-tuples of elements of $\Omega$, and suppose that $\left(Z_{1}, \ldots, Z_{q}\right) \sim \mu$. Then

$$
\|\mu-\nu\| \leq \sum_{l=0}^{q-1} \mathbf{E}\left(\left\|\mu\left(\cdot \mid Z_{1}, \ldots, Z_{\ell}\right)-\nu\left(\cdot \mid Z_{1}, \ldots, Z_{\ell}\right)\right\|\right) .
$$

Note that in the above lemma, since $Z_{1}, \ldots, Z_{q}$ are random variables (whose joint distribution is given by $\mu)$, so is $\left\|\mu\left(\cdot \mid Z_{1}, \ldots, Z_{\ell}\right)-\nu\left(\cdot \mid Z_{1}, \ldots, Z_{\ell}\right)\right\|$ for every $\ell<q$; each summand in the right-hand side of (2) is the expectation of one of these random variables.

Recall that $\tau_{t}^{k}$ is the conditional distribution of $X_{t}$ given $K_{1}, \ldots, K_{r}$. Fix $\ell \in$ $\{0, \ldots, q-1\}$. We wish to bound the expected distance between the distribution $\tau_{t}^{k}\left(\cdot \mid X_{t}(1), \ldots, X_{t}(\ell)\right)$ and $\pi\left(\cdot \mid X_{t}(1), \ldots, X_{t}(\ell)\right)$ (i.e., the uniform distribution on $\left.G \backslash\left\{X_{t}(1), \ldots, X_{t}(\ell)\right\}\right)$.

For $t \geq 0$, let $S_{t}=G \backslash\left\{X_{t}(1), \ldots, X_{t}(\ell)\right\}$. Thus $S_{t}$ is the set of positions that card $z_{\ell+1}$ could be located in at time $t$, given the positions of cards $z_{1}, \ldots, z_{\ell}$. For $a \in S_{t}$, let $p_{t}(a)=\tau_{t}^{k}\left(a \mid X_{t}(1), \ldots, X_{t}(\ell)\right)$. Then we have

$$
\left\|\tau_{t}^{k}\left(\cdot \mid X_{t}(1, \ldots, \ell)\right)-\pi\left(\cdot \mid X_{t}(1, \ldots, \ell)\right)\right\|=\frac{1}{2} \sum_{a \in S_{t}}\left|p_{t}(a)-1 / m\right|,
$$


where $m=\left|S_{t}\right|=N-\ell$. Using the Cauchy-Schwarz inequality twice gives

$$
\begin{aligned}
\left(\mathbf{E}\left[\sum_{a \in S_{t}}\left|p_{t}(a)-1 / m\right|\right]\right)^{2} & \leq \mathbf{E}\left[\left(\sum_{a \in S_{t}}\left|p_{t}(a)-1 / m\right|\right)^{2}\right] \\
& \leq m \cdot \mathbf{E}\left[\sum_{a \in S_{t}}\left(p_{t}(a)-1 / m\right)^{2}\right] \\
& \leq N \cdot \mathbf{E}\left[\sum_{a \in S_{t}}\left(p_{t}(a)-1 / m\right)^{2}\right] .
\end{aligned}
$$

We shall prove, by induction on $t$, that

$$
\mathbf{E}\left[\sum_{a \in S_{t}}\left(p_{t}(a)-1 / m\right)^{2}\right] \leq\left(\frac{\ell+N}{2 N}\right)^{t}
$$

for every $t \leq r$. Then, substituting $t=r$ to (3), (4), and (5), we have

$$
\begin{aligned}
& \mathbf{E}\left(\left\|\tau_{r}^{k}\left(\cdot \mid X_{r}(1, \ldots, \ell)\right)-\pi\left(\cdot \mid X_{r}(1, \ldots, \ell)\right)\right\|\right) \\
\leq & \frac{1}{2}\left(N \cdot \mathbf{E}\left[\sum_{a \in S_{r}}\left(p_{r}(a)-1 / m\right)^{2}\right]\right)^{1 / 2} \leq \frac{\sqrt{N}}{2}\left(\frac{\ell+N}{2 N}\right)^{r / 2} .
\end{aligned}
$$

Substituting this into Lemma 3 gives

$$
\begin{aligned}
\mathbf{E}\left(\left\|\tau_{r}^{k}-\pi\right\|\right) & \leq \sum_{\ell=0}^{q-1} \mathbf{E}\left(\left\|\tau_{r}^{k}\left(\cdot \mid X_{r}(1, \ldots, \ell)\right)-\pi\left(\cdot \mid X_{r}(1, \ldots, \ell)\right)\right\|\right) \\
& \leq \sum_{\ell=0}^{q-1} \frac{\sqrt{N}}{2}\left(\frac{\ell+N}{2 N}\right)^{r / 2} \\
& \leq N^{3 / 2} \int_{0}^{q / 2 N}(1 / 2+x)^{r / 2} d x \leq \frac{2 N^{3 / 2}}{r+2}\left(\frac{q+N}{2 N}\right)^{r / 2+1} .
\end{aligned}
$$

We now verify equation (5). First, consider the base case $t=0$. Since the initial positions of the cards are deterministic,

$$
\mathbf{E}\left[\sum_{a \in S_{0}}\left(p_{0}(a)-1 / m\right)^{2}\right]=(1-1 / m)^{2}+(m-1) \cdot(0-1 / m)^{2}=1-1 / m<1 .
$$

Now suppose that equation (5) holds for $t$. We prove that it also holds for $t+1$. Define $s_{t}=\sum_{a \in S_{t}}\left(p_{t}(a)-1 / m\right)^{2}$. It is sufficient to show that

$$
\mathbf{E}\left(s_{t+1} \mid s_{t}\right)=\left(\frac{\ell+N}{2 N}\right) s_{t} .
$$


Define $f: S_{t} \rightarrow S_{t+1}$ by

$$
f(a)= \begin{cases}a & \text { if } a \in S_{t+1} \\ K_{t+1}-a & \text { otherwise. }\end{cases}
$$

Note that $f$ is a bijection from $S_{t}$ to $S_{t+1}$ : it sends $S_{t}$ to $S_{t+1}$ because if $a \in S_{t}$ then either $a$ or $K_{t+1}-a$ must be in $S_{t+1}$, and it has an inverse $f^{-1}: S_{t+1} \rightarrow S_{t}$ defined by

$$
f^{-1}(b)= \begin{cases}b & \text { if } b \in S_{t} \\ K_{t+1}-b & \text { otherwise }\end{cases}
$$

Furthermore, note that

$$
p_{t+1}(f(a))= \begin{cases}p_{t}(a) & \text { if } K_{t+1}-a \notin S_{t} \\ \frac{1}{2} p_{t}(a)+\frac{1}{2} p_{t}\left(K_{t+1}-a\right) & \text { otherwise. }\end{cases}
$$

Since $K_{t+1}$ is independent of the process up to time $t$, for every $y \in G$, we have $\operatorname{Pr}\left[K_{t+1}-a=y \mid s_{t}\right]=1 / N$. Hence, since $\left|S_{t}\right|=m$, conditioning on the value of $K_{t+1}-a$ gives

$$
\mathbf{E}\left(\left[p_{t+1}(f(a))-\frac{1}{m}\right]^{2} \mid s_{t}\right)=\frac{\ell}{N}\left(p_{t}(a)-\frac{1}{m}\right)^{2}+\frac{1}{N} \sum_{y \in S_{t}}\left[\frac{p_{t}(a)+p_{t}(y)}{2}-\frac{1}{m}\right]^{2} .
$$

The sum can be rewritten as

$$
\begin{aligned}
& \sum_{y \in S_{t}} \frac{1}{4}\left[\left(p_{t}(y)-1 / m\right)+\left(p_{t}(a)-1 / m\right)\right]^{2} \\
= & \frac{1}{4} \sum_{y \in S_{t}}\left(p_{t}(y)-1 / m\right)^{2}+\frac{1}{2}\left(p_{t}(a)-1 / m\right) \sum_{y \in S_{t}}\left(p_{t}(y)-1 / m\right)+\frac{1}{4} \sum_{y \in S_{t}}\left(p_{t}(a)-1 / m\right)^{2} \\
= & \frac{1}{4} s_{t}+\frac{m}{4}\left(p_{t}(a)-1 / m\right)^{2},
\end{aligned}
$$

since $\sum_{y \in S_{t}}\left(p_{t}(y)-1 / m\right)=0$. Combining this with (7) gives

$$
\mathbf{E}\left(\left[p_{t+1}(f(a))-1 / m\right]^{2} \mid s_{t}\right)=\frac{s_{t}}{4 N}+\frac{4 \ell+m}{4 N}\left(p_{t}(a)-1 / m\right)^{2} .
$$

Note that

$$
\begin{aligned}
\mathbf{E}\left(s_{t+1} \mid s_{t}\right) & =\sum_{b \in S_{t+1}} \mathbf{E}\left(\left[p_{t+1}(b)-1 / m\right]^{2} \mid s_{t}\right) \\
& =\sum_{a \in S_{t}} \mathbf{E}\left(\left[p_{t+1}(f(a))-1 / m\right]^{2} \mid s_{t}\right) .
\end{aligned}
$$

Evaluating each term in the sum using (8) gives

$$
\begin{aligned}
\mathbf{E}\left(s_{t+1} \mid s_{t}\right) & =\frac{m s_{t}}{4 N}+\frac{4 \ell+m}{4 N} \sum_{a \in S_{t}}\left(p_{t}(a)-1 / m\right)^{2} \\
& =\frac{m s_{t}}{4 N}+\frac{(4 \ell+m) s_{t}}{4 N} \\
& =\frac{\ell+N}{2 N} s_{t},
\end{aligned}
$$


where the last line holds because $m+\ell=N$. It follows that $\mathbf{E}\left(s_{t+1} \mid s_{t}\right)=$ $\left(\frac{\ell+N}{2 N}\right) s_{t}$, which verifies (6) and hence (5). This completes the proof.

CCA-SECURITy. Observe that if $E=\mathrm{SN}[r, N,+]$ for some abelian group $G=$ $([N],+)$ then $E^{-1}$ is also $\mathrm{SN}[r, N,+]$. Employing Lemma1 we conclude our main theorem.

Theorem 4. Let $E=\operatorname{SN}[2 r, N,+]$. Then $\operatorname{Adv}_{E}^{\text {cca }}(q) \leq \frac{4 N^{3 / 2}}{r+2}\left(\frac{q+N}{2 N}\right)^{r / 2+1}$.

\section{Complexity-Theoretic Interpretation}

While Theorem 4 is information-theoretic, it should be clear that the result applies to the complexity-theoretic setting too, in exactly the same manner as Luby-Rackoff [14] and its successors. Namely, from a PRF $F: \mathcal{K} \times\{0,1\}^{*} \rightarrow$ $\{0,1\}$ and a number $n$, define $n$-bit round functions $F_{i}(X)$ whose $j$ th bit is $F(\langle i, j, n, X\rangle)$. Also define $n$-bit round keys $K_{i}$ whose $j$ th bit is $F(\langle i, j, n\rangle)$. Using these components, apply the swap-or-not construction for, say, $r=7 n$ rounds, yielding a PRP $E$ on $n$ bits. Translating the information-theoretic result into this setting, the PRP-security of $E$ is the PRF-security of $F$ minus a term that remains negligible until $q=(1-\epsilon) 2^{n}$ adversarial queries, for any $\epsilon>0$. That is, from the asymptotic point of view, the swap-or-not construction preserves essentially all of a PRF's security in the constructed PRP.

We emphasize that our security results only cover the (strong) PRP notion of security. An interesting question we leave open is whether the swap-or-not cipher is indifferentiable from a random permutation [16]. Following Coron, Patarin, and Seurin [6], Holenstein, Künzler, and Tessaro show that the 14-round Feistel construction is indifferentiable from a random permutation [12]. But their proof is complex and delivers very poor concrete-security bounds. It would be desirable to have a construction supporting a simpler proof with better bounds.

\section{Format-Preserving Encryption}

In the format-preserving encryption (FPE) problem, one wants to encipher on an arbitrary set $\mathcal{X}$, often $\mathcal{X}=[N]$ for some number $N$. Usually constructions are sought that start from a conventional blockcipher, like AES. The problem has attracted increasing interest [1 $5,5,8,9,17,24,25,27]$, and is the subject of ongoing standardization work by NIST and the IEEE.

When $N$ is sufficiently small that one can afford $\tilde{\Omega}(N)$-time to encrypt, provably good solutions are easy, by directly realizing a random shuffle [3]. And when $N$ is sufficiently large that no adversary could ask anything near $N^{1 / 2}$ queries, nice solutions are again easy, using standard cryptographic constructions 
like multi-round Feistel. But for intermediate-size domains, like those with $2^{30}-2^{60}$ points, the bounds associated to well-known construction are disappointing, even if known attacks are not remotely feasible, and spending time proportional to the domain size, even in key-setup phase, is not attractive.

With these problematic-size domains in mind, suppose we use swap-or-not to encipher 9-digit social security numbers $\left(N \approx 2^{30}\right)$. Employing Theorem 4 , if we use 340 rounds we are guaranteed a maximal CCA advantage of less than $10^{-10}$ even if the adversary can ask $q=10^{8}$ queries. Similarly, suppose we use swap-or-not to encipher 16 -digit credit cards $\left(N \approx 2^{53}\right)$. If we use 500 rounds we are guaranteed a maximal CCA advantage of less than $10^{-10}$ even if the adversary can ask $q=10^{15}$ queries. (Of course these numbers assume random round functions; if one bases the construction on AES, say, one will have to add in a term for its insecurity.) The round counts are obviously high, yet the rounds are fast and the guarantees are strong. (We note too that, at least for the binary-string setting and AES as a starting point, there are tricks to reduce the number of blockcipher calls by a factor of five, as shown in prior work [17]. But this is probably not helpful in the presence of good AES support, as with recent Intel processors.)

A very different approach to small-domain FPE is taken by Granboulan and Pornin [9], who show how to realize a particular shuffle on $N$ cards in $O\left(\lg ^{3} N\right)$ encryption time and $O(\lg N)$ space. But the method seems to be impractical, requiring extended-precision arithmetic to sample from a hypergeometric distribution. Stefanov and Shi go on to show how to exploit preprocessing to realize a different $N$-card shuffle [24]. Their method is applicable when the key-setup cost of $\tilde{\Theta}(N)$ is feasible, as is key storage and per-message encryption cost of $\tilde{\Theta}\left(N^{1 / 2}\right)$. Near or beyond $N \approx 2^{30}$, these assumptions seem unlikely to hold in most settings. That said, the approach allows an adversary to query all $N$ points, whereas the shuffle of this paper has only been proven to withstand $(1-\epsilon) N$ queries. (We conjecture that swap-or-not works well for $N$ queries and reasonable $r$ - that its mixing time is fast-but no such result is proven here.)

\section{Confusion/Diffusion Ciphers}

Swap-or-not can also be construed as an approach for making a confusion/diffusion blockcipher. In doing this one would instantiate round functions $F_{i}:\{0,1\}^{n} \rightarrow\{0,1\}$ by a fast, concrete construction. Perhaps the simplest plausible instantiation is have $F_{i}$ be specified by an $n$-bit

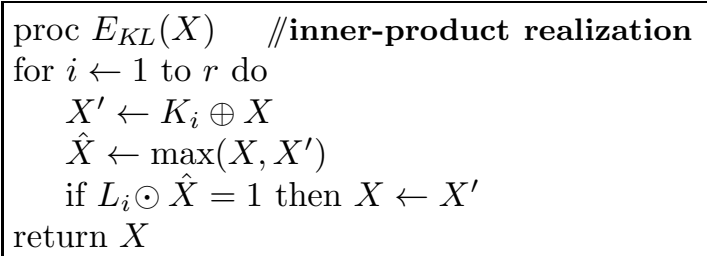

Fig. 5. Cipher $E=\mathrm{SN}[r, n, \odot]$ encrypts a string $X \in\{0,1\}^{n}$ using a key $K L$ that specifies subkeys $K_{1}, \ldots, K_{r}, L_{1}, \ldots, L_{r} \in\{0,1\}^{n}$ 
string $L_{i}$, letting $F_{i}(\hat{X})=L_{i} \odot \hat{X}=L_{i}[1] \hat{X}[1] \oplus \cdots \oplus L_{i}[n] \hat{X}[n]$ be the innerproduct of $L_{i}$ and $\hat{X}$. This concrete realization of swap-or-not is shown in Fig. 5 . (We comment that for this instantiation it is necessary to use "max" instead of "min" in selecting a canonical one of $\left\{X, X^{\prime}\right\}$; otherwise, we'd have $X=0^{n}$ always encrypting to $0^{n}$.)

We do not know how many rounds to suggest such that the construction of Fig. 5 should be a good blockcipher. It is incorrect to think that the theoretical analysis suggests a value like $r=6 n$; for one thing, there is an enormous gap between computing a random round function $F_{i}(\hat{X})$ and an inner product $L_{i} \odot \hat{X}$. We leave it as a problem for cryptanalysts to investigate how large $r$ needs to be, to ascertain if inner product with $L_{i}$ is actually a good choice for $F_{i}$, and to understand what other choices might work well.

Acknowledgments. The authors gratefully acknowledge comments from Mihir Bellare and Terence Spies. This work was supported under NSF grants DMS1007739 and CNS-0904380.

\section{References}

1. Bellare, M., Ristenpart, T., Rogaway, P., Stegers, T.: Format-Preserving Encryption. In: Jacobson Jr., M.J., Rijmen, V., Safavi-Naini, R. (eds.) SAC 2009. LNCS, vol. 5867, pp. 295-312. Springer, Heidelberg (2009)

2. Bellare, M., Rogaway, P., Spies, T.: The FFX mode of operation for formatpreserving encryption (February 2010) (submission to NIST, available from their website)

3. Black, J., Rogaway, P.: Ciphers with Arbitrary Finite Domains. In: Preneel, B. (ed.) CT-RSA 2002. LNCS, vol. 2271, pp. 114-130. Springer, Heidelberg (2002)

4. Brier, E., Peyrin, T., Stern, J.: BPS: a format-preserving encryption proposal (submission to NIST, available from their website)

5. Brightwell, M., Smith, H.: Using datatype-preserving encryption to enhance data warehouse security. In: 20th National Information Systems Security Conference Proceedings (NISSC), pp. 141-149 (1997)

6. Coron, J.-S., Patarin, J., Seurin, Y.: The Random Oracle Model and the Ideal Cipher Model Are Equivalent. In: Wagner, D. (ed.) CRYPTO 2008. LNCS, vol. 5157, pp. 1-20. Springer, Heidelberg (2008)

7. Diaconis, P., Fill, J.: Strong stationary times via a new form of duality. Annals of Probability 18(4), 1483-1522 (1990)

8. FIPS 74. U.S. National Bureau of Standards (U.S.). Guidelines for implementing and using the NBS Data Encryption Standard. U.S. Dept. of Commerce (1981)

9. Granboulan, L., Pornin, T.: Perfect Block Ciphers with Small Blocks. In: Biryukov, A. (ed.) FSE 2007. LNCS, vol. 4593, pp. 452-465. Springer, Heidelberg (2007)

10. Halevi, S.: EME*: Extending EME to Handle Arbitrary-Length Messages with Associated Data. In: Canteaut, A., Viswanathan, K. (eds.) INDOCRYPT 2004. LNCS, vol. 3348, pp. 315-327. Springer, Heidelberg (2004)

11. Halevi, S., Rogaway, P.: A Tweakable Enciphering Mode. In: Boneh, D. (ed.) CRYPTO 2003. LNCS, vol. 2729, pp. 482-499. Springer, Heidelberg (2003)

12. Holenstein, T., Künzler, R., Tessaro, S.: The equivalence of the random oracle model and the ideal cipher model, revisited. In: STOC 2011, pp. 89-98 (2011); Full version at arXiv:1011.1264 
13. Hoang, V.T., Rogaway, P.: On Generalized Feistel Networks. In: Rabin, T. (ed.) CRYPTO 2010. LNCS, vol. 6223, pp. 613-630. Springer, Heidelberg (2010)

14. Luby, M., Rackoff, C.: How to construct pseudorandom permutations from pseudorandom functions. SIAM J. on Computing 17(2), 373-386 (1988)

15. Maurer, U., Pietrzak, K., Renner, R.: Indistinguishability Amplification. In: Menezes, A. (ed.) CRYPTO 2007. LNCS, vol. 4622, pp. 130-149. Springer, Heidelberg (2007)

16. Maurer, U., Renner, R., Holenstein, C.: Indifferentiability, Impossibility Results on Reductions, and Applications to the Random Oracle Methodology. In: Naor, M. (ed.) TCC 2004. LNCS, vol. 2951, pp. 21-39. Springer, Heidelberg (2004)

17. Morris, B., Rogaway, P., Stegers, T.: How to Encipher Messages on a Small Domain: Deterministic Encryption and the Thorp Shuffle. In: Halevi, S. (ed.) CRYPTO 2009. LNCS, vol. 5677, pp. 286-302. Springer, Heidelberg (2009)

18. Naor, M., Reingold, O.: On the construction of pseudo-random permutations: Luby-Rackoff revisited. J. of Cryptology 12(1), 29-66 (1999)

19. Naor, M., Reingold, O.: A pseudo-random encryption mode (1997) (manuscript)

20. Patarin, J.: Pseudorandom Permutations Based on the DES Scheme. In: Charpin, P., Cohen, G. (eds.) EUROCODE 1990. LNCS, vol. 514, pp. 193-204. Springer, Heidelberg (1991)

21. Patarin, J.: Luby-Rackoff: 7 Rounds Are Enough for $2^{n(1-\varepsilon)}$ Security. In: Boneh, D. (ed.) CRYPTO 2003. LNCS, vol. 2729, pp. 513-529. Springer, Heidelberg (2003)

22. Patarin, J.: Security of balanced and unbalanced Feistel schemes with linear non equalities. Cryptology ePrint report 2010/293 (2010)

23. Rudich, S.: Limits on the provable consequences of one-way functions. Ph.D. Thesis, UC Berkeley (1989)

24. Stefanov, E., Shi, E.: FastPRP: Fast pseudo-random permutations for small domains. Cryptology ePrint Report 2012/254 (2012)

25. Stütz, T., Uhl, A.: Efficient Format-Compliant Encryption of Regular Languages: Block-Based Cycle-Walking. In: De Decker, B., Schaumüller-Bichl, I. (eds.) CMS 2010. LNCS, vol. 6109, pp. 81-92. Springer, Heidelberg (2010)

26. Thorp, E.: Nonrandom shuffling with applications to the game of Faro. Journal of the American Statistical Association 68, 842-847 (1973)

27. Wen, J., Severa, M., Zeng, W., Luttrell, M., Jin, W.: Circuits and systems for video technology. IEEE Transactions on Circuits \& Systems for Video Technology 12(6), 545-557 (2002) 